\title{
Radiologic Findings of Scapular Langerhans Cell Histiocytosis Successfully Treated with CT-Guided Corticosteroid Injection
}

\author{
Cennet Sahin ${ }^{1}$, Burcin Agridag Ucpinar ${ }^{1}$, Yunus $\mathrm{Oc}^{2}$ and Fevziye Kabukcuoglu ${ }^{3}$ \\ ${ }^{1}$ Department of Radiology, University of Health Sciences, Istanbul Sisli Hamidiye Etfal Training and Research Hospital, Istanbul, Turkey \\ ${ }^{2}$ Department of Orthopaedic Surgery, TekirdagCorlu Vega Hospital, Turkey \\ ${ }^{3}$ Department of Pathology, University of Health Sciences, Istanbul Sisli Hamidiye Etfal Training and Research Hospital, Istanbul, Turkey
}

\begin{abstract}
Although Langerhans cell histiocytosis (LCH) may arise from any bone, flat bones (skull, ribs, scapula, clavicle, and mandible) are more commonly involved.The overall reported incidence of scapular involvement by LCH is nearly $3 \%$ in the literature. Intralesional corticosteroid injection can be given for lesions that cause pain or postural deformity. We, herein, report a 26-year male with scapular LCH who was suffering from right shoulder pain and right arm weakness. The patient received intra-lesional methylprednisolone under CT-guidance for treatment. On follow-up imaging, almost total regression was observed. We suggest that percutaneous CT-guided intra-lesional steroid injection is an efficient method for the treatment of LCH located in bone.
\end{abstract}

Key Words: Bone, Langerhans cell histiocytosis, Local treatment, Corticosteroid.

How to cite this article: Sahin C, Ucpinar BA, Oc Y, Kabukcuoglu F. Radiologic Findings of Scapular Langerhans Cell Histiocytosis Successfully Treated with CT-Guided Corticosteroid Injection. J Coll Physicians Surg Pak 2020; 30(07):754-756.

\section{INTRODUCTION}

Langerhans cell histiocytosis ( $\mathrm{LCH}$ ) is a disorder characterised by clonal proliferation of Langerhans cells. ${ }^{1,2}$ Isolated form affects a certain area of the body (mostly bones), which is named as eosinophilic granuloma. ${ }^{1,2}$ Solitary form is more common than disease affecting multiple sites and has a better prognosis. ${ }^{3}$ While multi-organ LCH may require anti-cancer chemotherapy and other systemic therapies, intra-lesional steroid injection is one of the treatment options that may be curative for solitary form of $\mathrm{LCH} .{ }^{4-6} \mathrm{Although}$, efficacy and safety of the intra-lesional steroid injection in LCH has been reported in many studies, very few of them included radiological cross-sectional follow-up imaging pre-and post-treatment.

In this study, we aim to present cross-sectional computed tomography (CT) and magnetic resonance imaging (MRI) findings of a scapular LCH, diagnosed with CT-guided percutaneous biopsy and successfully treated with intra-lesional methylprednisolone injection.

Correspondence to: Burcin Agridag Ucpinar, Department of Radiology, University of Health Sciences, Istanbul Sisli Hamidiye Etfal Training and Research Hospital, Istanbul, Turkey

E-mail: drburcinagridag@gmail.com

Received: July 26, 2019; Revised: September 23, 2019;

Accepted: October 01, 2019

DOI: https://doi.org/10.29271/jcpsp.2020.07.754

\section{CASE REPORT}

A 26-year male patient, who had been suffering from right shoulder pain and arm weakness for the last 3 months, was referred to our hospital. A complete blood count, and blood chemistry analyses were performed, which were unremarkable, except for a slight increase in leukocytes and C-reactive protein levels. Shoulder X-ray was performed for the first radiologic evaluation. Since there was an oval shaped radiolucent area on the radiograph (Figure $1 \mathrm{~A}$ ), shoulder MRI was performed for further evaluation. A $30 \times 20 \mathrm{~mm}$ diameter osteolytic lesion with well-defined borders at the level of scapula was seen on $\mathrm{T} 1$ and $\mathrm{T} 2$-weighted images (Figures 1B, 1C). There was perilesional soft tissue edema on T2-weighted images. To establish a better evaluation, a targeted CT was performed regarding high resolution of the $\mathrm{CT}$ in the evaluation of bony structures. Multiplanar CT examination better demonstrated the lesion with well-defined borders (Figure 1D). The lesion was mildly expansile with periosteal reaction and had a sclerotic area in the centre.

To establish the final diagnosis, CT-guided percutaneous bone biopsy was performed after taking informed consent. Under CTguidance (Siemens, Somotom, Emotion; Germany), core biopsy and fine-needle aspiration biopsy were obtained. The histopathological examination revealed large oval mononuclear cells with few cytoplasmic vacuoles and a prominent nucleus. Immunohistochemically, the cells were positive for the cluster designation 1a (CD1a) and S-100 (Figure 2). The microbiological examination did not reveal any infectious process. Since the 
patient had only shoulder pain, intra-lesional steroid injection was planned for local treatment. Under local anesthesia and sterile conditions, $125 \mathrm{mg}$ methylprednisolone was injected into the lesion through a 20-g Chiba-needle under CT guidance.

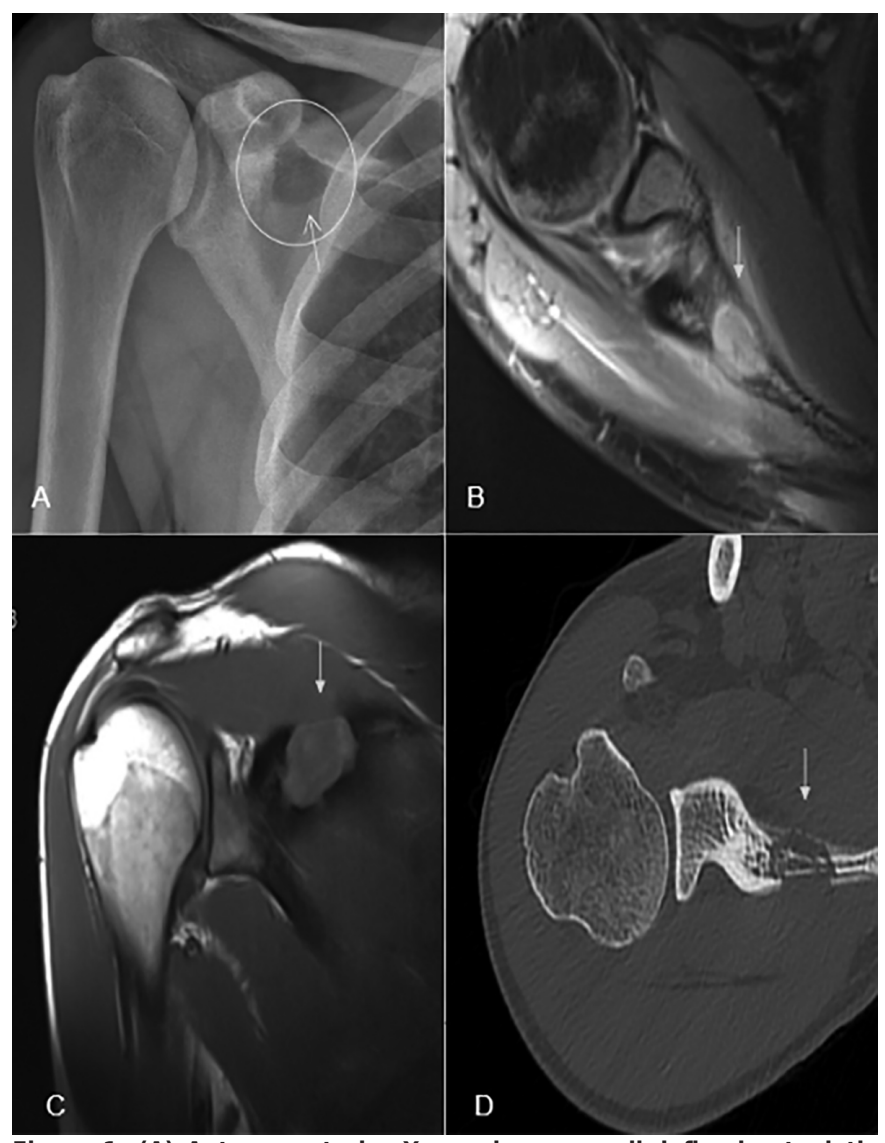

Figure 1: (A) Antero-posterior X-ray shows a well-defined osteolytic lesion (marked) in the scapula. (B) Axial MR image of scapula shows well-circumscribedlesion and perilesional soft tissue edema which is hyperintense on T2-weighted image. (C) The lesion is seen hypointense on coronal T1-weighted image. (D) Axial CT shows punched out lytic lesion on the scapular spine. Sclerotic area in the lesion denotes dense sclerotic central focus, which corresponds to button sequestrum of the lesion. Lucent area represents the erosive accumulation of histiocytes.

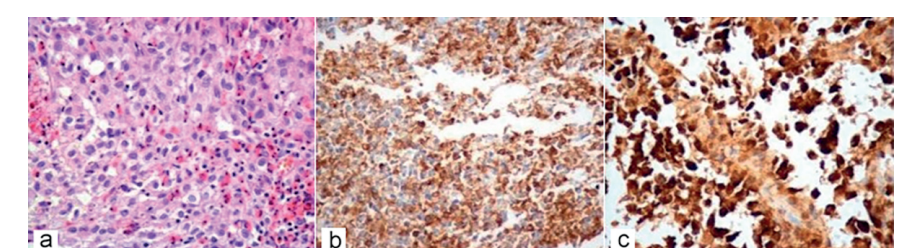
and a prominent nucleus were shown with arrows. Many eosinophils are also present. (hematoxylin and eosin stain; magnification $\times 40$ ). (B) Immunohistochemically, the cells were positive for the cluster designation 1a (CD1a) (magnification $\times 40$ ). (C) The cells were also positive forS-100 (magnification $\times 40$ ). CD1a and S-100 are histiocytic markers and the brown color indicates positive reaction.

The patient had a complete pain relief within 48 hours after procedure. After four weeks on routine follow up, he had neither pain complaint, nor arm weakness. To determine the effect of local steroid treatment radiologically, follow-up MRI was performed on the $4^{\text {th }}$ week, which showed diminution in intensity of the lesion on T2-weighted images due to bone reconstruc- tion; while, the lesion volume was still the same (Figure. 3A). On the $7^{\text {th }}$ week after the initial treatment, pain complaint relapsed without concomitant arm weakness. A second steroid injection was planned. After the second injection, the pain relieved within 48 hours again. On the $33^{\text {rd }}$ week after the initial treatment, follow-up cross-sectional MRI (Figure 3B) revealed almost entire regression of the lesion. The patient was pain-free and did not have any complaint.

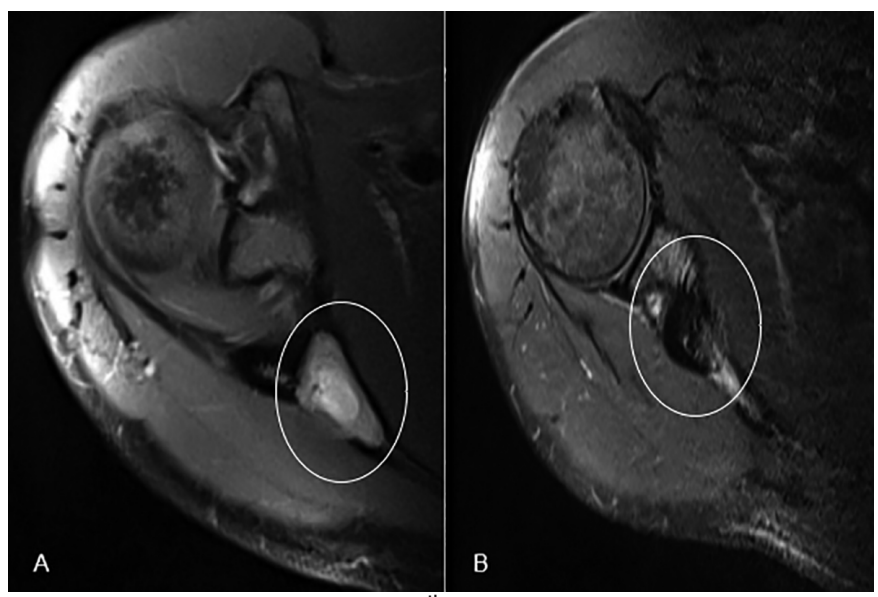

Figure 3: (A) Follow-up MRI on the $4^{\text {th }}$ week after steroid injection showed decrement in intensity of the lesion (red circle) on T2-weighted images, probably due to bony reconstruction while the lesion volume was still the same. (B) The non-contrastT2-weighted image of the follow-upcross-sectional MRI on $33^{\text {th }}$ week after the first treatment revealed almost total regression of the scapularlesion.

\section{DISCUSSION}

Although LCH may arise from any bone, flat bones (skull, ribs, scapula, clavicle, and mandible) are generally more commonly involved. ${ }^{1,2}$ The overall reported incidence of scapular involvement of LCH is nearly $3 \%$ in the literature. ${ }^{5,6}$

LCH of bone may present with pain, soft tissue swelling, limping of the extremity, postural disability and fever. ${ }^{3}$ Although shoulder pain and arm weakness were the only symptoms in our patient, he had a diagnostic work-up with positron emission tomography computerised tomography (PET-CT) for a possible systemic involvement; and no other organ involvement was observed.

On X-rays, bone lesions may present as intramedullary lytic lesions. ${ }^{2}$ Benign periosteal reaction may also be seen. Calvarial lesions can be observed as "button sequestrum" or as multiple "punched-out" lytic lesions. ${ }^{2}$ Vertebral lesions can be seen as vertebra plana (flattened vertebrae) with/without kyphosis. ${ }^{6} \mathrm{MRI}$ imaging better demonstrates the medullary extent of the lesion and soft tissue edema; while CT better identifies the intra-lesional sclerosis and periosteal reaction. ${ }^{5} \mathrm{LCH}$ can mimic malignancy, especially when it presents as solitary bone lesion. ${ }^{6}$ PET-CT may help to show if any organ involvement is present. On the other hand, PET-CT may give false-negative results in older lesions. ${ }^{1,5}$ Correlation between cross-sectional imaging and histopathological examination are needed as a rule.

Treatment strategy of LCH depends on the extent and severity of the disease at onset. ${ }^{3}$ Main objectives of the treatment are to 
diminish pain and to prevent possible complications, such as bone fracture related to lytic lesions. ${ }^{6}$ Since spontaneous remission may occur in the solitary self-limited disease, observation alone is the most preferred strategy in such cases, especially in the pediatric age goup. ${ }^{3}$ Intra-lesional corticosteroid injection or curettage of the isolated bony lesion can be performed for lesions that cause pain or postural deformity like kyphosis. ${ }^{3,5}$ Low dose irradiation may be preferred for lesions that are located in inaccessiblesites. Percutaneous radiofrequencyablation is another treatment modality reported in the literature. In severe cases, such as disease with extraskeletal involvement and the disease with two or more organ involvement, intensive chemotherapy combinations like MACOP-B (methotrexate with leucovorin rescue, doxorubicin, cyclophosphamide, vincristine, prednisone, and bleomycin) can be preferred as treatment strategy. ${ }^{6,7}$

Radiological healing of eosinophilic granuloma may take a long time. ${ }^{2}$ While clinical response and pain relief may be observed in 48 hours, radiological healing time may range between 3 to 24 months. ${ }^{4}$ In our case, the patient had a rapid pain relief in 48 hours after intra-lesional steroid injection therapies. In the first 7 weeks after the initial steroid injection, the radiological response was very slow. After the second therapy, radiological response was faster than the first. Since, there is no standard protocol for radiological follow-up in these patients, we had our own protocol for follow-up, depending on the clinical and radiological conditions.

In conclusion, intra-lesional steroid injection is minimally invasive procedure when compared with intraoperative curettage and also a curative treatment option. Total or nearly-total radiological response can be shown in follow-up cross-sectional CT and MRI.

\section{PATIENTS' CONSENT:}

Written informed consent was obtained from the patient.

\section{CONFLICT OF INTEREST:}

The authors declared no conflict of interest.

\section{AUTHORS' CONTRIBUTION:}

CS: Data acquisition and analysis, interpretation, drafting and final approval.

BAU: Conception and design, Interpretation, drafting and final approval.

YO, FK: Critical revision, final drafting and final approval.

\section{REFERENCES}

1. Stull MA, Kransdorf MJ, Devaney KO. Langerhans cell histiocytosis of bone. Radiographics 1992; 12(4):801-23.

2. Azouz EM, Saigal G, Rodriguez MM, Podda A. Langerhans cell histiocytosis: Pathology imaging and treatment of skeletal involvement. PediatrRadiol 2005; 35:103-15.

3. Girschikofsky M, Arico M, Castillo D, Chu A, Doberauer C, Fichter J, et al. Management of adult patients with Langerhans cell histiocytosis: Recommendations from an expert panel on behalf of Euro-Histio-Net. Orphanet J Rare Dis 2013; 8:72.

4. Cohen M, Zornoza J, Cangir A, Murray JA, Wallace S. Direct injection of methylprednisolone sodium succinate in the treatment of solitary eosinophilic granuloma of bone: $A$ report of 9 cases. Radiology 1980; 136(2):289-93.

5. Zaveri J, La Q, Yarmish G, Neuman J. More than just langerhans cell histiocytosis. A radiologic review of histiocytic disorders. Radiographics 2014; 34(7):2008-25.

6. Angelini A, Mavrogenis AF, Rimondi E, Rossi G, Ruggieri P. Current concepts for the diagnosis and management of eosinophilic granuloma of bone. J OrthopTraumatol 2017; 18(2):83-90.

7. Derenzini E, Fina MP, Stefoni $V$, Pellegrini $C$, Venturini $F$, Broccoli $A$, et al. MACOP-B regimen in the treatment of adult Langerhans cell histiocytosis: Experience on seven patients. Ann Oncol 2010; 21(6):1173-8. 